\title{
RICHARD Nicolás (ed.), Mala guerra. Los indígenas en la guerra del Chaco (1932-1935)
}

\section{Capucine Boidin}

\section{OpenEdition}

\section{Journals}

Édition électronique

URL : https://journals.openedition.org/jsa/10818

DOI : 10.4000/jsa. 10818

ISSN : 1957-7842

\section{Éditeur}

Société des américanistes

\section{Édition imprimée}

Date de publication : 23 juillet 2009

Pagination : 243-247

ISSN : 0037-9174

\section{Référence électronique}

Capucine Boidin, « RICHARD Nicolás (ed.), Mala guerra. Los indígenas en la guerra del Chaco

(1932-1935) », Journal de la Société des américanistes [En ligne], 95-1 | 2009, mis en ligne le 10 juillet 2009, consulté le 04 septembre 2022. URL : http://journals.openedition.org/jsa/10818 ; DOI : https:// doi.org/10.4000/jsa. 10818

Ce document a été généré automatiquement le 4 septembre 2022

Tous droits réservés 


\title{
RICHARD Nicolás (ed.), Mala guerra. Los indígenas en la guerra del Chaco (1932-1935)
}

\author{
Capucine Boidin
}

\section{RÉFÉRENCE}

RICHARD Nicolás (ed.), Mala guerra. Los indígenas en la guerra del Chaco (1932-1935), ServiLibro-Museo del Barro/CoLibris, Asunción/Paris, 2008, 421 p., bibl., cartes

L'ouvrage s'inscrit dans une dynamique de recherche collective trinationale (française, bolivienne et paraguayenne), commencée depuis 2004 et qui a récemment pris la forme d'un projet ANR «Les Indiens dans la Guerre du Chaco » (2006-2010). Une substantielle introduction pluridisciplinaire de 52 pages, intitulée Histoire d'une absence et anthropologie d'un oubli, est co-signée par Nicolás Richard (ethnologue, Chaco paraguayen), Isabelle Combès (ethnohistorienne, Chaco bolivien) et Luc Capdevila (historien, Paraguay). Elle donne les résultats des premières enquêtes de terrain et d'archives pour restituer la présence et les paroles indigènes tout en analysant les conditions de production de leur oubli. Une bibliographie générale de 26 pages ainsi que cinq cartes sont de précieux outils de recherche. Les treize textes permettent de dessiner un panorama général du Chaco, organisé en différentes zones de guerre et zones ethniques : les articles de Rodrigo Villagra ainsi que d'Ernesto Unruh et Hannes Kalish traitent du Chaco central paraguayen habité par les Enlhet-enenlhet. Lorena Cordoba et José Braunstein comme Miguel Fritz abordent la zone du Pilcomayo, argentine et paraguayenne, habitée par les Toba et les Chulupi dits aussi Niwaklé. Barbara Schuchard, Jürgen Riester, Isabelle Combès, Diego Villar, Federico Bossert et Eric Langer explorent la situation sur la marge occidentale et bolivienne du Chaco, habitée par les Guarani Chané et Chiriguano. Edgardo J. Cordeu, Nicolás Richard et Volker von Bremen reprennent le problème du Chaco septentrional et l'Alto Paraguay où dominent les Chamacoco et les Ayoré. L'article de 
José Zanardini recompose l'ensemble de manière diachronique pour la partie paraguayenne après la guerre, incluant en particulier un point sur les Maka. Le dernier texte de Maria de Fatima Costa aborde une période plus éloignée, en montrant les dynamiques des guerres antérieures.

2 L'introduction est particulièrement centrale pour tous ceux qui cherchent à comprendre le double processus selon lequel les indigènes deviennent les subalternes des histoires nationales et les essences réifiées des anthropologies nationales et internationales. La manière dont l'historiographie bolivienne et paraguayenne dépouille les indigènes du statut de sujet dialogique - les privant littéralement de parole - est exemplaire. Elle constituerait une annexe à la fameuse déconstruction que réalise Gayatri Chakravorty Spivak concernant la subalternisation des femmes indiennes, excepté que, dans ce volume, nous avons à la fois l'analyse de la production du silence et l'émergence de la parole. Nous aurions pu choisir un autre axe de lecture comme celui des conséquences de la guerre sur les réorganisations des territorialités indigènes, mais il nous semble que les différents statuts donnés à la parole indigène explorés ici constituent un fil directeur heuristique pour des chercheurs spécialistes d'autres régions et thématiques.

\section{De la production du silence... Naturaliser l'Indien}

Si la bibliographie sur cette guerre de la soif et du désert qui opposa la Bolivie et le Paraguay de 1932 à 1935 est abondante, elle reste centrée sur les éléments logistiques, militaires, diplomatiques et parfois sociaux du conflit. Le théâtre des opérations a été vidé de ses milliers d'indigènes, par l'imagination comme par la réalité des violences de guerre. Les écrits sur la guerre font systématiquement comme si le Chaco n'était habité que par les mennonites, installés de fait depuis peu, ou bien procèdent par approximation, réduisant la multiplicité des groupes linguistiques à l'une ou l'autre ethnie plus connue. Les histoires nationalistes de la guerre mentionnent très peu leur présence et lorsqu'elles les mettent en scène, elles les renvoient au statut de simple élément de décor naturel. Au mieux, une certaine fascination admirative peut parfois se faire sentir, en particulier pour la virilité des hommes et la nudité des femmes, devenue érotique dans leur regard... Naturalisés, non considérés a priori comme sujets/acteurs politiques, les Indiens laissent peu de trace dans les archives et les mémoires. Pourtant, les armées ont largement dépendu du système d'occupation du territoire pour localiser les points d'eau et choisir les emplacements de fortins. Elles ont engagé aussi dans leurs troupes des éclaireurs et des espions indigènes. Parfois elles les ont armés. Et ces derniers ont transmis aux générations suivantes leurs mises en mots et mémoires de l'événement. Mais il fallait pour les pays impliqués donner à voir une guerre «blanchie » et moderne, gommant la parole indienne comme les processus coloniaux et colonisateurs de ce conflit.

Parallèlement, l'anthropologie classique du Chaco accorde peu de place à cet événement historique dans la structuration des différents groupes ethniques, bien qu'il soit à l'origine de redéploiements démographiques et politiques considérables. Deshistoricisés, les indigènes du Chaco pouvaient ainsi apparaître dans toute leur virginité, naturelle, primitive. Alors même que les premières monographies anthropologiques sont rendues possibles par la guerre et écrites dans la foulée des bottes des soldats, elles oblitèrent systématiquement la présence des chars, camions, fusils et fortins qui entourent les campements qu'ils pénètrent et étudient. Alfred Métraux, par exemple, 
publie en 1933 un article sur les guerres indiennes où mythes, rites et scalps emplissent les pages sans aucune mention des fusils ou chars qui le rendent possible. En oblitérant ainsi ses conditions de production, l'anthropologie ne manifeste que mieux son lien intime avec le projet de colonisation nationale. Même si certains épisodes de la guerre donnent le premier rôle aux Indiens, en réalité ils sont considérés comme anecdotiques et ne prennent pas de valeur anthropologique. La situation commence à changer à partir des années 1980, sous l'impulsion de deux facteurs: la nécessité pour la génération de la guerre de laisser un témoignage à celle qui est née dans les missions ou les estancias - en raison de la guerre précisément ; un tournant dans la discipline anthropologique où la parole indienne devient centrale.

\section{... aux logiques, sociologies et mémoires indiennes}

5 En quoi le volume permet-il d'aller au-delà de ces quelques histoires paradigmatiques qui n'émergent que dans la mesure où elles servent la mémoire nationale ou missionaire? Une première piste consiste à explorer les logiques indiennes de la guerre : Isabelle Combès, par exemple, nous restitue comment l'histoire de Casiano Barrientos un chef chiriguano bolivien retourné ou fait prisonnier (selon les interprétations) par les militaires paraguayens - peut être réécrite à partir des logiques internes d'affrontements politiques. L'auteur nous montre également que « la guerre n'a pas provoqué de réponses absolument neuves mais a plutôt renforcé des dynamiques sociales préexistentes : au lieu des migrations partielles et temporaires se mettent en place des migrations définitives pour des communautés entières; au lieu d'exiler des chefs de clan indésirables, ils sont envoyés se faire fusiller » (Combès, p. 230).

6 Nicolás Richard va dans le même sens lorsqu'il reconstitue la sociologie et les logiques propres des baqueanos chamacoco qui ont guidé l'explorateur russe Belaïeff, lequel travaillait pour le compte du Paraguay. Richard nous montre comment les guides sont des individus marginalisés (captifs, métis, étrangers) au sein de groupes déjà rattachés aux périphéries des ports fluviaux distribués le long du fleuve Paraguay : se mettre au service de l'explorateur russe qui les armait et leur donnait un cheval, leur permettait alors de se libérer de leur statut marginal, de devenir du même coup caciques de ces tribus tout en retrouvant leur groupe d'origine situé dans l'intérieur du Chaco. Dans tous les cas, la présence de Belaïeff et du régiment altère la relation entre les colons des ports fluviaux et les indigènes : les Indiens sont, en effet, considérés comme plus stratégiques pour les armées que les colons eux-mêmes. Au point que Belaïeff propose de constituer une cavalerie chamacoco. Le projet sera rejeté, mais les Chamacoco deviennent des sujets et des interlocuteurs pour Belaïeff, avec lesquels il noue des liens d'amitié et de confiance.

7 Une deuxième piste consiste à prendre les témoignages indiens comme des sources historiographiques en tant que telles et non pas seulement pour reconstituer «leur vision» de la guerre. En ce sens, l'article de Cordeu est probablement le plus fascinant parce qu'il joint une réflexion philosophique à des données très précises : il ne veut pas se contenter de dessiner le sens de l'événement pour les Ishir (même s'il termine en précisant que, pour les Ishir, les avions furent identifiés à des personnes, avant d'être interprétés comme des machines), il cherche à conjuguer mémoire indigène et archives pour reconstituer précisément ce qui s'est passé: trajet des avions, nombre de bombes larguées, mort de tel ou tel officier. Les concordances entre témoignages et archives sont 
rares, parce que la mémoire indigène rapporte davantage de détails d'événements liés à un ici et maintenant qu'elle ne propose un récit articulant un avant et un après (Cordeu, p. 287). Sa stratégie d'écriture est intéressante : il commence par juxtaposer huit récitssynthèses d'entretiens où, à côté de récits plus classiques de massacre, d'épidémie, de résistance aux vaccins, d'avions assimilés à des esprits-monstres, l'on découvre des femmes qui transportaient et enfouissaient des jarres d'eau et des hommes qui gagnaient des grades de capitaines en trouvant des points d'eau grâce à leurs savoirs chamaniques ou en prenant les armes plus courageusement que des officiers paraguayens. Puis il analyse les archives à sa disposition, pour enfin réunir les deux dans des tableaux synoptiques (Cordeu, pp. 283, 285).

\section{... et aux paroles indiennes : « nous n'étions pas des ennemis »}

8 Cet ouvrage donne surtout accès aux réflexions développées par des invidus et des groupes à propos de leur exclusion de tout échange verbal et armé. Les récits enlhet rappellent, par exemple, que les États en conflit se sont disputé une terre qui ne leur appartenait pas, et qu'eux-mêmes, véritables maîtres des lieux, ne furent pas perçus comme des ennemis (Unruh et Kalish, p. 100). Pour des sociétés où les identités masculines se constituent précisément par la guerre, il est paradoxal et cruel de ne pas les avoir considérées comme les parties adverses du conflit. Les Guarani Chiriguano vont plus loin: c'est parce que les Boliviens n'ont pas su reconnaître et utiliser les compétences guerrières des Chiriguano qu'ils ont perdu la guerre. « À ce moment-là, les Blancs (karai) ne nous considéraient pas comme des humains mais comme des animaux. Malgré cela, ils nous ont fait participer au conflit de différentes manières " (Riester, p.190). En effet, ils ne reçurent pas d'uniforme et furent simplement recrutés pour terrasser les chemins, épier l'ennemi, trouver les points d'eaux, guider les troupes ou enterrer les morts. "Imaginez un peu, si les Blancs nous avaient considérés comme Boliviens dès le départ, nous n'aurions jamais perdu. Parce que, nous, nous étions plus valeureux » (Riester, p. 194).

9 Les témoignages des Angaite sont d'une autre teneur, liés à leur actuel état de dépendance envers les missions. Ils identifient le temps de la guerre comme celui des soldats en colère kempohakme, avec lesquels ils étaient entrés en relation par l'intermédiaire des estancieros pour lesquels ils travaillaient déjà comme journaliers. Ils racontent avoir été sauvés des soldats en colère grâce à un missionaire anglais $\mathrm{El} \mathrm{gringo}$ nos salvó (Villagra, p. 92). Ce récit classique, presque devenu mythique dans certains cas, se répète pour les Chulupi-Niwaklé, « sauvés » par la mission des oblats allemands (Fritz, p. 153), ainsi que pour les Enhlet-enenhlet qui disent des mennonites : «Savhongvay': nos han ayudado " (Unruh et Kalish, p. 120). Mais les Angaité racontent aussi des histoires moins pré-formatées où domine l'absence de communication verbale entre Indiens et soldats. Ce sont des trocs sans paroles: des patates douces locales contre la yerba maté et les biscuits apportés par des soldats paraguayens. " Nos grands-pères ne savaient pas quels types de personnes étaient les soldats, il n'y avait pas de communication [...]. Avant, nos grands-pères ne parlaient que leur langue. Nous étions perdus dans l'erreur, ensuite nous avons appris le guarani » (Villagra, pp. 82-83).

10 Ainsi, cet ouvrage tout en déconstruisant l'invisibilisation et la réduction au silence des Indiens est en quelque sorte performatif: il les constitue en tant que sujet dialogique. 
Aussi pouvons-nous le recommander, au-delà des spécialistes du Chaco, à des chercheurs et des étudiants en histoire et en anthropologie, en particulier ceux qui s'intéressent aux articulations entre Indiens/Sociétés/États-Nations/Histoire/Guerre.

\section{AUTEURS}

\section{CAPUCINE BOIDIN}

IHEAL-Paris III Sorbonne Nouvelle, CREDAL 